\title{
Nutzenmaximierung - ein notwendiges, aber nicht hinreichendes Prinzip
}

\author{
Sollen oder dürfen bei der Festlegung der Grenzen sozial finanzierter Gesundheits- \\ leistungen Kosten-Nutzen-Überlegungen berücksichtigt werden? In egalitaristischen \\ und nutzenorientierten Ethikansätzen wird dies unterschiedlich eingeschätzt. Im \\ Myozyme-Urteil wird Stellung bezogen, aber die Umsetzung bleibt unklar.
}

Markus Zimmermann-Acklin

Korrespondenz:

PD Dr. theol. Markus Zimmermann-Acklin Dept. für Moraltheologie und Ethik

Theologische Fakultät

Universität Freiburg

Avenue de l'Europe 20

CH-1700 Freiburg

markus.zimmermann@unifr.ch
Eine heikle Frage, die durch das sogenannte MorbusPompe-Urteil des Bundesgerichts [1] aufgeworfen wird, lautet: Sollen oder dürfen bei der Festlegung der Grenzen sozial finanzierter Gesundheitsleistungen Kosten-Nutzen-Überlegungen und damit Maximierungsstrategien berücksichtigt werden? Sollen oder dürfen die Ressourcen im Gesundheitswesen so eingesetzt werden, dass sie einen optimalen Nutzen erbringen? Aus gesundheitsethischer Sicht wird damit eine Forderung problematisiert, die aus gesundheitsökonomischer Sicht trivial klingt.

\section{Nutzenmaximierung als Kriterium umstritten}

In der Ethik gehen die Meinungen hier auseinander. Das mag an der unterschiedlichen Einschätzung der Frage liegen, inwieweit Prinzipien und Methoden der Ökonomik ethisch überhaupt berücksichtigt werden sollten. Ethik und Ökonomik sind traditionell Konkurrentinnen, und in den Rationierungsdebatten treffen sie gleichsam direkt aufeinander und ringen um den Primat. Das Grundprinzip der Ökonomik besteht in der Anerkennung der Knappheit aller Mittel, dasjenige der Ethik in der Anerkennung der Würde aller Menschen. Das Ziel beider Handlungstheorien besteht in einer möglichst gerechten Verteilung der Ressourcen. Dazu knüpft die (Werte-)Ökonomik methodisch an der Nutzenoptimierung, die Ethik an der Anerkennung von Rechten und Pflichten an.

Inwieweit aus Sicht der Ethik die Verständigung zwischen beiden Disziplinen gesucht wird, ist an der gewählten Ethiktheorie erkennbar: Es geht um den Konflikt zwischen egalitaristischen (auf Gleichbehandlung aller beruhenden, kantianisch geprägten) und nutzenorientierten (z. B. utilitaristischen) Theorieansätzen. Egalitaristische Ethiken berücksichtigen ausschliesslich Rechte, Ansprüche und Pflichten und beruhen auf der Anerkennung des Prinzips der allen Menschen gleichermassen zukommenden Würde. Utilitaristische Ethiken beurteilen Handlungen dagegen primär anhand ihrer Folgen und anerkennen die Nutzenmaximierung als zentrales Ethikkriterium. Der klassische Utilitarismus ist nicht zufällig im England

\section{Maximisation de l'utilité -}

\section{un principe nécessaire}

\section{mais insuffisant}

Doit-on ou peut-on tenir compte du rapport coûtutilité lors de la détermination du plafond de prise en charge des prestations de santé par l'assurance sociale? Les approches éthiques égalitaires, axées sur l'efficacité ne s'accordent pas toutes. En effet, l'approche empirique considère autant l'aspect de l'égalité de traitement que celui de son efficacité, ce qui n'est pas sans conséquences, étant donné que la prise en compte de l'efficacité des coûts peut occasionner des inégalités de traitement problématiques. Si les juges fédéraux estiment qu'une utilité plus élevée justifie également des coûts plus élevés, cela peut paraître convaincant sur le principe. En revanche, sur le terrain - comme dans le cas du médicament orphelin Myozime justement- il faut aussi tenir compte des circonstances. L'arrêt du Tribunal fédéral montre certes la voie à suivre en indiquant qu'on devrait prendre en compte les réflexions sur le rapport coût-utilité, mais les juges restent muets quant à la manière d'appliquer ce principe.

der Industrialisierung und des aufkommenden Kapitalismus entstanden und verdankt diesem wesentliche Impulse.

Eine kürzlich erschienene Stellungnahme des Deutschen Ethikrats ist hilfreich, um diesen Disput im Hinblick auf die Verteilungsdebatten besser einordnen zu können [2]. Weyma Lübbe lehnt darin in einem ausführlichen Sondervotum Maximierungsstrategien grundsätzlich ab, da damit nicht nur 
Urteile über bestimmte Behandlungen abgegeben würden, sondern gleichzeitig auch Urteile über bestimmte Patienten: "Nach hier vertretener Auffassung sind solche Urteile nicht vertretbar. Unvertretbar ist daran nicht, dass am Ende nicht allen alles erstattet wird. Unvertretbar ist die Art der Begründung. Sie unterstellt, Entscheidungen öffentlicher Instanzen über den Ausschluss bestimmter Leistungen seien dadurch begründbar, dass bestimmte (nützliche) Leistungen es «nicht wert sind, finanziert $\mathrm{zu}$ werden. Für die Betroffenen mag das, wie man weiss, ganz anders aussehen. Aus der Sicht eines Krebspatienten, der den Kampf gegen die Krankheit noch nicht aufgegeben hat, kann sich die Alternative von Erstattung und Nichterstattung wie die Alternative von Sein oder Nichtsein anfühlen. (...) Wenn nun amtlich festgestellt wird, das Medikament habe keinen hinreichenden Wert, dann bleibt gar nichts übrig, als dies so zu hören, dass die Gesellschaft keinen hinreichenden Wert mehr auf das weitere Überleben der Betroffenen legt.» [2, S. 115 f]. Für Weyma Lübbe sind solche Missverständnisse der Grund dafür, den Diskurs anders zu führen, und zwar ausschliesslich über Rechte und legitime Ansprüche der Einzelnen. «Man muss vielmehr plausibel machen, dass es angesichts der knappen Mittel ungerecht wäre, den Versicherten einen Anspruch auf diese Leistung zuzugestehen.» $[2$, S. $116 \mathrm{f}]$.

\section{Kosteneffektivität: ein Kriterium neben anderen}

Ein erfahrungsnaher Zugang zur ethischen Beurteilung der Verteilungsprobleme kommt nicht nur zu einem ähnlichen Ergebnis, sondern gewichtet sowohl

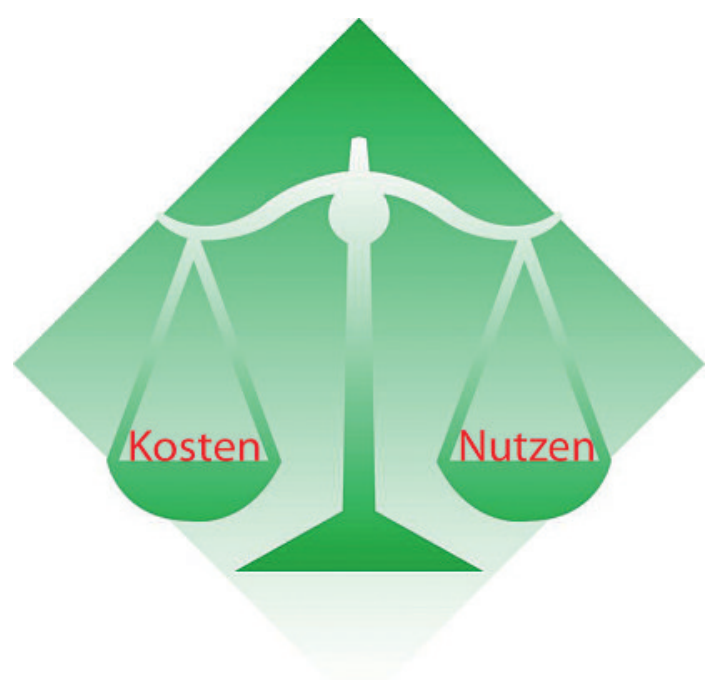

In der Ethik gehen die Meinungen darüber auseinander, welche Rolle Kosten-Nutzen-Erwägungen im Gesundheitswesen spielen dürfen.

lung aller bleibt eine regulative Idee, die in der Praxis alltäglichen Handelns leitend sein soll, aber praktisch nie erreicht werden kann. Dies kommt z.B. in der «Rule of rescue» zum Ausdruck, die im Sinne des Bedürfnis- und Solidaritätsprinzips davon ausgeht, dass besonders gefährdetes Leben ganz besondere Aufmerksamkeit erhalten sollte. Das britische National Institute for Health and Clinical Excellence (NICE) hat an diesen Stellen «Korrekturen von Hand» an ihren Kosten-Nutzen-Berechnungen angebracht, insofern sie die monetären Schwellenwerte für einen

\section{Die Idee, qualitätsbereinigte Lebensjahre (QALYs) in Geldeinheiten auszudrücken, ist mit einer ganzen Reihe von Problemen verbunden.}

egalitaristische als auch Nutzenüberlegungen. Intuitiv scheint zuzutreffen: Es kann gerechter sein, bei der Zuteilung knapper Ressourcen Unterschiede zwischen Menschen zuzulassen, die auf Nutzenüberlegungen beruhen [3]: eine Einsicht, die mit steigendem Ausmass von Knappheit auch auf zunehmende gesellschaftliche Akzeptanz stossen dürfte. Wichtig ist, dass jede Entscheidung zugunsten der Finanzierung einer Massnahme stets eine Entscheidung darüber mit beinhaltet, welche anderen Massnahmen stattdessen unterlassen werden. Vorgegeben ist, dass wir uns stets schon in einem System der Zuteilung befinden, in dem entschieden wird und werden muss, welchen Nutzen wir hervorbringen möchten und welchen nicht.

Die Orientierung an der Kosteneffektivität alleine wäre allerdings nicht vertretbar. Die Gleichbehand-
QALY (ein qualitätsbereinigtes Lebensjahr) bei Sterbenden und Menschen mit seltenen Krankheiten markant erhöht hat [4, S. 20 f].

\section{QALYs als Lösung?}

Werden Kosten-Nutzen-Überlegungen in ein ethisches Kalkül aufgenommen, sind schwierige Folgeprobleme zu lösen. Diese betreffen das Verhältnis dieser Nutzenüberlegungen zu den Grundrechten, sie betreffen aber auch Fragen der Kosteneffektivitätsberechnungen, da insbesondere die Idee, qualitätsbereinigte Lebensjahre (QALYs) in Geldeinheiten auszudrücken, mit einer ganzen Reihe von Schwierigkeiten umzugehen hat. Das zeigt bereits der Begriff «Qualität» an, der individuell sehr unterschiedlich beurteilt wird: Mögliche Nebenwirkungen der Behandlung eines Prostatakarzinoms wie Inkontinenz 
oder Impotenz beispielsweise dürften nicht von allen Betroffenen gleichermassen eingeschätzt werden.

Die QALYs sind zudem «blind» für die Frage, wie schwer ein Mensch von einer Einschränkung betroffen ist. Inhärent werden ältere Menschen, Menschen mit Behinderungen oder auch Patienten mit seltenen lungskosten für eine äusserst seltene Erkrankung oder eine weit verbreitete Tumorerkrankung beurteilt werden. Behandlungskosten bei Ultra-Orphan-Drugs wie Myozyme liegen in der Regel viel höher als Kosten für häufig praktizierte Standardbehandlungen, Studien zum Nachweis von deren Nutzen sind aufgrund der

\section{Die Gleichbehandlung aller bleibt eine regulative Idee, die in der Praxis alltäglichen Handelns leitend sein soll, aber praktisch nie erreicht werden kann.}

Erkrankungen benachteiligt, letztere deshalb, weil entsprechende Medikamente in der Regel sehr teuer sind. Eine ungelöste Frage besteht zudem in der Festlegung von Schwellenwerten, welche angeben, wie viel ein QALY kosten darf, um sozial finanziert zu werden [3, S. $220 \mathrm{f}]$.

Diese Probleme weisen zum einen darauf hin, dass die QALYs alleine keine Handhabe zur ethischen Bewertung bieten, sondern andere Kriterien voraussetzen [4]. Zum anderen wird deutlich, dass die Kosteneffektivität als Rationierungskriterium die teils hohen Erwartungen nicht wirklich erfüllen kann.

\section{Das Morbus-Pompe-Urteil: richtungsweisend,} aber systematisch ungenügend

In der Begründung des Morbus-Pompe-Entscheids fällt auf, dass Kosten-Nutzen-Angaben undifferenziert eingeführt werden. Nur an einer Stelle im Urteil ist von QALYs die Rede, die häufig zitierten 100000 Franken dagegen werden auf ein gerettetes Menschenjahr bezogen, also ein Zeitmass ohne Relation zur Lebensqualität.

Als Kriterium geben die Bundesrichter die Verhältnismässigkeit der Kosten an: «Je höher der Nutzen, desto höhere Kosten sind gerechtfertigt.» [1, Abs. 7.4] Als Grundsatz mag das überzeugen, allerdings müssen bei dessen Anwendung die Umstände berücksichtigt werden. So ist wesentlich, ob Behand- kleinen Zahl Betroffener nur bedingt aussagekräftig [5]. Daneben wären Qualitätsstandards einzuführen und monetär zu gewichten und nicht zuletzt Schwellenwerte politisch zu legitimieren.

In diesem Sinne kann das Urteil zwar als richtungsweisend hinsichtlich der Aussage gelten, dass Kosten-Nutzen-Überlegungen berücksichtigt werden sollten, systematisch aber lässt es uns weitgehend im Stich.

Literatur

1 BG 9C_334/2010 vom 23.11.2010.

2 Deutscher Ethikrat. Nutzen und Kosten im Gesundheitswesen - Zur normativen Funktion ihrer Bewertung. Stellungnahme. Berlin; 2011.

3 Marckmann G. Kosteneffektivität als Allokationskriterium aus gesundheitsethischer Sicht. In: Zimmermann-Acklin M, Halter H (Hrsg.). Rationierung und Gerechtigkeit im Gesundheitswesen. Beiträge zur Debatte in der Schweiz. Basel: EMH; 2007. S. 213-24.

4 National Institute for Health and Clinical Excellence, Social Value Judgements. Principles for the development of NICE guidance. Second Edition. 2008. www.nice.org.uk (11.7.2011).

5 Van der Ploeg AT et al. A Randomized Study of Alglucosidase Alfa in Late-Onset Pompe's Disease. New Engl J Med. 2010;362:1396-406. 